\title{
Cent ans de l'enseignement de la linguistique romane à la Faculté de philosophie et lettres de Zagreb
}

\author{
August Kovačec \\ Sveučilište u Zagrebu \\ august.kovacec@inet.hr
}

Après la réorganisation et la modernisation de l'Université de Zagreb, c'est à partir de l'année 1882 qu'a été introduit, à la Faculté des lettres et philosophie, l'enseignement pratique du français et peu après de l'italien. Cependant ce n'est qu'en 1919 qu'a été créé, auprès de la Faculté, un Département (/Séminaire) de philologie romane. Le premier professeur de linguistique romane a été Petar Skok, ancien élève de Wilhelm Mayer-Lübke à l'Université de Vienne. Skok a entrepris des recherches systématiques sur les éléments romans de la côte orientale de l'Adriatique et des pays balkaniques. Petar Guberina, un des élèves de Skok, a introduit dans l'enseignement de la linguistique les points de vue et les méthodes de l'école de Genève, avant tout la stylistique de Ch. Bally. Le premier professeur de philologie italienne a été Mirko Deanović qui a étudié les emprunts aux langues romanes dans la terminologie maritime de la côte adriatique et a proposé l'élaboration d'un atlas linguistique des langues de la Méditerranée. À partir des années 1960, c'est Vojmir Vinja qui a conduit le Département de philologie romane. Il a étudié systématiquement les éléments romans empruntés par les parlers croates de la côte et des îles ainsi que la toponymie d'origine romane. Ce sont Vinja et Deanović qui ont encouragé les jeunes romanistes d'entreprendre une description systématique des idiomes romans parlés sur le territoire de la Croatie et de la Bosnie. C'est grâce aux efforts de Vojmir Vinja qu'à la Faculté des lettres ont été organisées des chaires pour d'autres langues romanes que le français et l'italien. Aujourd'hui, les activités des romanistes de la Faculté des lettres, dans le domaine de la linguistique, sont organisées en cinq sections - française, espagnole, portugaise, roumaine et italienne - au sein de deux départements, le Département de philologie romane et le Département de philologie italienne.

Mots-clés : balkaniques (langues), calque, constructions navales, dalmate, emprunts, étymologie, istriote (istro-roman), istroroumain, judéo-espagnol, stylistique, terminologie maritime, terminologie de pêche, toponymie, vénitien

Avant de passer à la présentation des études linguistiques au Département des langues et littératures romanes au cours de ces cent dernières années, je voudrais remercier M. Nikica Talan, professeur de langue et littérature portugaises, pour avoir préparé, il y a quelques années, une histoire détaillée de l'évolution des études romanes à la Faculté de philosophie et lettres de 
l’Université Zagreb depuis leur mise en place en 1884. Je tiens à remercier aussi mes collègues $\mathrm{M}^{\text {mes }}$ Sanja Šoštarić et Marija Paprašarovski qui ont complété et mis à jour les données réunies par M. Talan. Je les remercie tous trois d'avoir mis leur texte à ma disposition.

Ensuite, je voudrais souligner qu'à leurs débuts, les chaires de langue et de littérature italiennes faisaient partie du Département (Séminaire) roman, après quoi elles ont été organisées en Département de langue et littérature italiennes. Cette autonomie administrative n'empêcha toutefois pas les deux départements de continuer à fonctionner, sur le plan des études linguistiques, en plein accord et en parfaite collaboration. Toute présentation des activités du Département roman qui omettrait de mentionner les activités du Département italien serait donc incomplète, et c'est pourquoi je vais aussi prendre en considération les études linguistiques du Département italien.

Dans cette présentation, je vais parler tout d'abord de l'activité des linguistes qui ont mené à terme leurs activités à la Faculté et ne mentionnerai que quelques noms de ceux qui, parmi mes consœurs et confrères, sont toujours actifs dans l'enseignement. Je ne mentionnerai qu'en passant les spécialistes qui ont consacré leurs activités à l'étude de la littérature.

Les Croates ont établi des contacts avec les populations romanophones dès une époque très ancienne et leurs relations mutuelles - politiques, sociales, culturelles - se sont poursuivies pendant des siècles. Il faut tenir compte avant tout du fait que deux langues romanes se sont formées sur le territoire de la Croatie actuelle : le dalmate, langue romane autochtone de quelques villes côtières et insulaires de Dalmatie, et la langue istriote ou istro-romane, présente dans quelques villes et bourgades de la partie sud-ouest de l'Istrie. Au cours des siècles, la population rurale croatophone a pénétré progressivement dans les villes de Dalmatie et l'idiome roman autochtone s'est éteint avec le temps. À Dubrovnik, le dalmate ragusain s'est maintenu en usage, semble-t-il, jusqu'au XV siècle, tandis que le dernier locuteur du dalmate de la ville de Krk, dans l'île du même nom (Veglia en italien), est mort au cours de la dernière décennie du XIX ${ }^{\mathrm{e}}$ siècle. Par ailleurs, au cours du Moyen-Âge, les populations de l'intérieur de la péninsule balkanique, dont une partie au moins parlait une variété du roumain, se sont déplacées vers la côte dalmate et l'Istrie. La pénétration vénitienne vers $l^{\prime}$ Istrie et les côtes orientales de l'Adriatique a débuté dès le $X^{\mathrm{e}}$ siècle et, jusqu'au $\mathrm{XV}^{\mathrm{e}}$ siècle, la République de Venise a assis sa domination en Dalmatie ainsi que dans une grande partie de l'Istrie. Après s'être imposé en tant que langue internationale de commerce maritime et langue de l'administration de l'État, l'italien s'est maintenu en usage, à côté du latin, en Dalmatie et en Istrie pendant des siècles, non seulement en tant que langue de l'administration, mais aussi comme langue de communication quotidienne d'une partie de la population urbaine. Parallèlement, dès le Moyen-Âge, nombre de jeunes gens non seulement de Dalmatie mais aussi ceux des autres régions de la Croatie actuelle, faisaient 
leurs études universitaires dans des universités italiennes et françaises. Tous ces faits expliquent l'intérêt pour l'enseignement et l'étude des langues romanes en Croatie.

Quelques années après la réorganisation et la modernisation de l'Université de Zagreb et après la fondation d'une Faculté de philosophie et lettres en 1874, des cours pratiques des langues romanes ont été mis en place dans le cadre de cette dernière, mais il a fallu attendre plusieurs décennies avant de pouvoir organiser un enseignement et des études modernes et complètes des langues et littératures romanes. D'abord, au cours de l'année académique 1883/1884, ont été organisés des cours de grammaire française ainsi que des cours consacrés à l'analyse de telle ou telle œuvre littéraire ou de tel et tel écrivain français, au rythme de quelques heures par semaine. C'est en 1889/1890 que l'enseignement de la langue et littérature françaises a été confié à Julije Adamović, qui s'efforcera de présenter dans ses cours, outre l'enseignement pratique du français, l'ensemble des problèmes de la grammaire française et de donner un panorama de la littérature française. Adamović a enseigné jusqu'en 1926. Il a signé, entre autres, plusieurs manuels de français, des livres de lectures, un important dictionnaire français-croate (Adamović, 1901; 1921), mais il est surtout l'auteur d'une excellente Grammaire française (écrite en croate) qui, jusqu'à la Deuxième Guerre mondiale, a été utilisée comme manuel de base pour l'enseignement du français dans les écoles (Adamović, 1893). Dès la fin du XIX ${ }^{\mathrm{e}}$ siècle, la Faculté de philosophie et lettres a également organisé en son sein des cours de langue italienne.

Une nouvelle orientation dans l'évolution des études romanes à l'Université de Zagreb se fait jour en 1913, lorsque Petar Skok obtient le droit d'enseigner la grammaire historique des langues romanes à l'Université. Tout d'abord, il donne un cours traitant des éléments et de la génèse du latin vulgaire ainsi qu'un cours sur les emprunts romans en croate. En 1915/1916, Petar Skok commence à enseigner la grammaire historique de la langue française et, l'année académique suivante, la grammaire historique de l'italien. En 1919, Petar Skok est nommé professeur de philologie romane et, un an plus tard, en 1920, la Faculté de philosophie et lettres se dote d'un Séminaire (Département) de philologie romane comportant une section française et une section italienne. Le professeur Skok y a enseigné jusqu'à sa retraite en 1952, avec une interruption de deux ans pendant la guerre (1943-1945) (Vinja, 1981; Kovačec, 1993; Muljačić, 1995).

Petar Skok fit ses études de philologie germanique et de philologie romane de 1900 à 1904 à l'Université de Vienne, où il étudia aussi la philologie slave et la grammaire comparée des langues indoeuropéennes. C'est à la même université qu'il prépara, sous la direction de Wilhelm Mayer-Lübke, une thèse de doctorat traitant des toponymes formés à l'aide des suffixes -ācum, -ānum, -ascum et -uscum en France méridionale. Il soutint sa thèse en 1905, et la publia en 1906 (Skok, 1906). Cette thèse fait toujours autorité dans les études de la toponymie sur le territoire de la France. Avant d'être nommé professeur, Skok rédigea toute une série de travaux importants traitant de questions linguistiques, avant tout étymologiques, des langues romanes, du latin, de l'illyrien et du croate. On peut mentionner, à titre d'exemple, son livre sur les phénomènes du latin vulgaire 
dans les inscriptions de la province romaine de Dalmatie (Skok, 1915). Entre les deux guerres mondiales, le professeur Skok publia une série de manuels universitaires (comme par exemple un manuel de linguistique romane en trois volumes /Skok, 1940/, un aperçu de grammaire française /Skok, 1938; Skok, 1939/ etc.). À la même période il fit paraître dans des revues prestigieuses un grand nombre d'études sur la symbiose romano-slave et les contacts romano-slaves en Dalmatie et dans la péninsule balkanique (Skok, 1928; 1935), sur la terminologie maritime et de la pêche du littoral adriatique, sur des questions de toponymie etc. Il faut mentionner tout particulièrement ses études sur le dalmate (Skok, 1926; 1927) et l'istriote (Skok, 1936a; 1943), ainsi que ses travaux de balkanologie (Skok, 1929; 1936), marqués par un intérêt prononcé pour le roumain et l'albanais (Skok, 1926a), ainsi que pour l'influence turque sur les langues balkaniques. Dès ses premiers travaux de linguistique, Skok s'intéressa tout particulièrement à l'étymologie. En 1943, le professeur Skok fut mis en retraite et c'est Slavko Ježić (1895-1969) qui fut nommé professeur de philologie romane. Slavko Ježić a fait ses études de philologie romane et de philologie slave à Vienne où il a soutenu sa thèse de doctorat en 1916. Éminent historien de la littérature et critique littéraire (qui traduisit aussi en croate une série de chef-d'œuvres de différentes langues) Ježić est aussi l'auteur d'une excellente histoire de la littérature française de 842 à 1715 (Ježić, 1928). Au cours des années de sa retraite forcée, le professeur Skok entreprit de compléter systématiquement son fichier étymologique dans le but de composer et d'élaborer un dictionnaire étymologique de la langue croate. Bien que ce dictionnaire n'ait pas pu être terminé par son auteur (Skok mourut en 1956) et que ses quatre volumes aient été achevés et publiés par un des élèves et des confrères du professeur, il représente une œuvre capitale dans le domaine de l'étymologie sud-slave, balkanique et romane (Skok, 1971; 1972; 1973; 1974; Kovačec, 1976). Romaniste et balkanologue, linguiste disposant de connaissances profondes dans le domaine de l'albanologie, de la byzantologie, de la turcologie etc., Skok s'efforça non seulement de donner l'étymologie des éléments lexicaux du croate littéraire, mais aussi celle de toutes les variétés dialectales des territoires croates, bosniaques, monténégrins et serbes, en prêtant une attention particulière aux éléments lexicaux empruntés aux langues romanes, au grec, à l'albanais, au turc. Après la guerre, Skok publia des travaux sur les contacts linguistiques romano-slaves tels qu'ils se reflètent dans la toponymie de la côte orientale de l'Adriatique et des travaux sur l'istriote, de même que sur la conquête de Zadar par les croisés au XIII ${ }^{\mathrm{e}}$ siècle ainsi qu'elle se reflète dans les chroniques françaises du Moyen-Âge. Outre des cours de linguistique, le professeur Skok donnait aussi des cours de littérature française. De temps à autre, la littérature française était également enseignée par les lecteurs français venus de France.

Comme nous l'avons déjà dit, on donnait des cours pratiques de langue italienne à la Faculté de philosophie et lettres depuis la fin du XIX ${ }^{\mathrm{e}}$ siècle. C'est en 1934 que, dans le cadre du Séminaire roman, a été fondée une chaire de langue italienne. Bien qu'elle ait été organisée à partir de 1940 en Séminaire (Département) italien, administrativement indépendant du Séminaire roman, les deux départements ont toujours collaboré. Le premier enseignant de philologie 
italienne fut Mirko Deanović (1890-1984). Après avoir étudié la philologie romane et la philologie slave à Florence et Vienne, et après avoir soutenu une thèse de doctorat à l'Université de Vienne, Mirko Deanović fut chargé de l'enseignement de la langue et littérature italiennes à partir de 1940 en qualité de professeur titulaire (Spomenica, 1986). Le professeur Deanović étudiait surtout les éléments romans qui ont pénétré dans les parlers croates de la côte, avant tout dans la terminologie maritime, ainsi que les contacts culturels et littéraires entre la France et l'Italie d'un côté, et Dubrovnik de l'autre. S'appuyant sur des enquêtes de terrain, le professeur Deanović publia une description du parler istriote de Rovinj (Deanović, 1954; cf. aussi Deanović, 1954a). En collaboration avec son confrère Josip Jernej, plus tard professeur titulaire au Département d'italien (Kovačec, 2005; 2012), Deanović publia un solide dictionnaire italien-croate (Deanović-Jernej, 1960) puis un dictionnaire croate-italien (Deanović-Jernej, 1956). Jernej est également, entre autres, l'auteur d'une remarquable grammaire descriptive et pratique de la langue italienne (Jernej, 1987). Mirko Deanović a aussi le mérite d'avoir proposé l'élaboration d'un Atlas linguistique méditerranéen et d'avoir mis en place une collaboration internationale, grâce avant tout aux institutions italiennes, en vue de la réalisation de ce projet. Le professeur Deanović stimulait et soutenait les recherches des jeunes linguistes sur les idiomes romans de la côte et des îles adriatiques, ainsi que les recherches sur les enclaves linguistiques romanes de l'arrière-pays. À partir des années 1960, la littérature italienne a été enseignée avant tout par d'anciens élèves du professeur Deanović: Mate Zorić et Frano Ćale.

Quant aux études romanes et françaises, l'œuvre de Skok a été poursuivie par Petar Guberina (1912-2005), l'un de ses meilleurs élèves. Petar Guberina termine ses études de philologie romane en 1935 à l'Université de Zagreb. À partir de 1937, il travaille comme assistant du professeur Skok au Séminaire de philologie romane. Parallèlement, le jeune Guberina continue ses études à la Sorbonne sous la direction de Pierre Fouché, dans le domaine de la phonétique, et sous la direction de Jules Marouzeau en linguistique générale et philologie classique (Filipović, 1987-1988; Kovačec, 2006; 2014). En 1939 Guberina soutient à la Sorbonne sa thèse de doctorat, dans laquelle il traite de la valeur logique et la valeur stylistique des propositions complexes en français et en croate selon, d'une part, les principes théoriques fondamentaux de Ferdinand Brunot et, de l'autre, les principes de la stylistique et de la linguistique du discours de Charles Bally (Guberina, 1939). Au lieu de l'analyse formaliste et positiviste de la proposition dictée par la syntaxe traditionnelle, Guberina propose dans sa thèse une analyse plus subtile, beaucoup plus proche des réalités de la communication. Cette analyse prend comme point de départ la proposition en tant que signe linguistique complexe, en tant qu'ensemble du contenu logique et des valeurs affectives. Guberina souligne également l'opposition entre langue écrite et langue parlée, en insistant sur le fait que, dans la forme parlée de la langue, qui représente sa forme primaire, agissent un certain nombre d'éléments extralinguistiques et paralinguistiques, à savoir de phénomènes tels que le contexte, la situation, l'intonation, le rythme, la pause etc., qui complètent la communication linguistique. Guberina fait également ressortir que, dans une analyse syntaxique, il est important d'examiner 
non seulement la proposition en elle-même, en tant qu'unité la plus élevée du point de vue hiérarchique, mais aussi de prendre en considération l'ensemble linguistique - le passage, le texte - dans lequel elle fonctionne. Par ailleurs, les contacts qu'établit Guberina avec d'éminents linguistes français lui permirent de parfaire également sa connaissance d'une partie des nouveaux courants de la linguistique européenne de la première moitié du XXe siècle. Rentré à Zagreb, Guberina publia plusieurs études traitant les questions de stylistique, la différence entre langue parlée et langue écrite. Malheureusement, l'éclatement de la guerre fit que sa thèse ne trouva pas, du moins en Croatie, l'écho qu'elle méritait. En 1943, le jeune linguiste interrompit sa carrière universitaire. Elle reprit en 1951 lorsque, le professeur Skok ayant été mis à la retraite, Guberina prit en charge l'enseignement de la linguistique romane et de l'histoire de la langue française. En 1954 il est nommé chef du Département de philologie romane. Au cours de cette période, il publie plusieurs livres et études par lesquels il applique à l'analyse linguistique les principes de l'École de Genève - notamment ceux de Ch. Bally, A. Sèchehaye et F. de Saussure (Guberina, 1952; 1952a). Il s'efforce aussi d'introduire ces méthodes dans l'enseignement en attachant une importance particulière à l'enseignement de la stylistique. Cependant, de retour à l'Université, le professeur Guberina n'y poursuit pas longtemps sa carrière de professeur de linguistique romane. Dès 1954, il fonde au sein de la Faculté de philosophie et lettres l'Institut de phonétique qui deviendra un peu plus tard le Département de phonétique. Bien que continuant, jusqu'au milieu des années 1960 (plus précisément jusqu'en 1964/65), à donner des cours de stylistique pour les étudiants de français, il se consacra surtout aux recherches dans le domaine de la phonétique expérimentale et à l'enseignement de la phonétique, à l'étude de l'audition ainsi qu'à la recherche sur différents aspects de l'acte de la parole et sur l'élaboration d'une nouvelle méthode d'apprentissage des langues étrangères. À la faveur de ces recherches nécessairement interdisciplinaires, le professeur Guberina développa une collaboration étroite avec des physiciens de l'acoustique, des médecins spécialistes en otorhinolaryngologie, des orthophonistes etc., ce qui contribua à l'éloigner peu à peu du champ de la philologie romane proprement dite.

Plus ou moins à l'époque où le professeur Guberina fait son retour à la Faculté, son ancien confrère Antun Polanšćak (1910-1978), qui a soutenu à la Sorbonne une thèse sur Marcel Proust (en 1941), va à partir de 1952 se charger de l'enseignement de la littérature française. Cette activité sera poursuivie plus tard par ses élèves, Predrag Matvejević, Jelica Arneri Vidan, Jere Tarle, Ingrid Šafranek, puis par Nenad Ivić, Maja Zorica et autres.

C'est en 1958/1959 que la direction du Département de philologie romane, après avoir été assumée par le professeur Guberina, fut reprise par Vojmir Vinja (1921-2007). Lui aussi élève de Petar Skok, Vinja termina ses études de philologie romane en 1947, et peu après se vit élire assistant au Département de philologie romane (Kovačec, 2008; 2008a; 2010; 2013). Sa thèse de doctorat, soutenue en 1952, était consacrée aux éléments d'origine romane dans les parlers de l'île de Korčula. Vinja enseignait avant tout la linguistique romane et l'histoire de la langue française, mais il accordait aussi une importance particulière à l'enseignement 
de la linguistique générale, notamment de ses courants modernes. Vojmir Vinja fut le premier à organiser au sein de la Faculté de philosophie et lettres un enseignement systématique de la linguistique saussurienne et de la linguistique structurale en général, y compris plus particulièrement l'enseignement de la sémantique moderne. En tant que chef du Département de philologie romane, le professeur Vinja s'efforça dès la fin des années 1950, d'assurer un enseignement régulier des langues et littératures romanes autres que les langues et littératures françaises et italiennes. Il veilla également à ce que les cadres nécessaires pour asseoir cette nouvelle orientation reçoivent une formation adéquate. Tout d'abord, il fit organiser périodiquement des cours pratiques de portugais, d'espagnol et de roumain. Il prépara et publia lui-même une grammaire de la langue espagnole et un grand dictionnaire espagnol-croate, ce qui rendit possible l'organisation de cours pratiques permanents d'espagnol puis, plus tard, l'organisation d'études complètes de cette langue dans le cadre de la Chaire de langue et littérature espagnoles. Il était convaincu, tout comme ses professeurs Skok et Deanović, que la tâche principale de nos romanistes n'est pas seulement d'étudier les grandes langues et grandes littératures romanes, mais aussi d'étudier systématiquement, d'un côté, les langues romanes autochtones sur le territoire croate et sud-slave en général (comme le dalmate et l'istriote) et, de l'autre, les relations entre les Croates et les peuples romans sur le plan littéraire et culturel. C'est avant tout grâce aux efforts du professeur Vinja que commença à fonctionner, à partir de la fin des années 1960, une chaire de langue et littérature espagnoles puis, à partir des années 1980, une chaire de langue et littérature portugaises et enfin, dès les premières années de notre siècle, une chaire de langue et littérature roumaines. Depuis le commencement de sa carrière universitaire jusqu'à sa mise à la retraite en 1986, le professeur Vinja mena des enquêtes systématiques, fondées sur les méthodes de la géographie linguistique, dans presque 200 localités de la côte orientale et dans les îles de l'Adriatique pour y recueillir un matériel linguistique contenant des éléments romans (essentiellement des termes maritimes et de pêche, des mots relatifs à la flore et la faune maritimes, à la toponymie etc.). Au cours des années il consacra aux éléments romans (d'origine dalmate et d'origine italienne) dans les parlers croates une série d'études dans lesquelles il se penche particulièrement sur les problèmes étymologiques et sémantiques. Enfin, il a publié deux ouvrages synthétiques sur les dénominations de la faune adriatique (Vinja, 1986) et sur les étymologies et la sémantique du „vocabulaire adriatique" (Vinja, 1998; 2003, 2004; 2016; Kovačec, 2005a), complément important au Dictionnaire étymologique de Skok.

Comme nous l'avons déjà dit, les professeurs Guberina, Deanović et Vinja attribuaient une grande importance à l'enseignement pratique des langues romanes. À côté des lecteurs français et italiens, qui enseignaient ces deux langues dès la période de l'entre-deux-guerres, ils engagèrent un certain nombre de maîtres de langue croates, possédant une connaissance pratique des langues romanes, et dont certains se sont particulièrement distingués dans cette activité.

Au cours des années 1960, les professeurs Deanović et Vinja ont pareillement encouragé et motivé leurs confrères, surtout les plus jeunes, à entreprendre une 
description systématique des idiomes des enclaves romanes sur le territoire de la Croatie. Ainsi, Domenico Cernecca a commencé à étudier son parler istro-roman natal de Bale (it. Valle). Pavao Tekavčić a donné une description structuraliste précise du parler istro-roman de Vodnjan (it. Dignano) et a continué, jusqu'à la fin de ses jours, à étudier les questions relatives aux idiomes istriotes (Kovačec, 2007). Conscient de l'importance des contacts linguistiques, Tekavčić envisageait l'élaboration d'un atlas linguistique de l'Istrie. Malheureusement, à l'époque cette idée n'a pas pu être réalisée, mais à partir des années 1990 c'est le professeur Goran Filipi de l'Université de Pula (it. Pola) qui l'accomplira en réunissant, grâce à des enquêtes sur le terrain basées sur un questionnaire, un matériel linguistique pour tous les idiomes d'Istrie (trois groupes de parlers croates, istro-vénitien, istriote, istro-roumain, monténégrin, slovène) qui sera publié en sept volumes. L'istro-roumain est étudié, à partir des années 1960, par A. Kovačec (Kovačec, 1971 ; 1998) qui scrute également les vestiges des parlers judéo-espagnols de Dubrovnik et de Sarajevo. Pavao Tekavčić est quant à lui l'auteur d'un grand nombre d'études traitant de l'histoire de la langue italienne, de la dialectologie italienne, des idiomes rhéto-romans etc. Par ailleurs, un des plus grands mérites de Tekavčić est d'avoir rédigé et publié une solide introduction au latin vulgaire (Tekavčić, 1970) ainsi qu'une introduction à la linguistique générale destinée aux italianistes et aux romanistes en général (Tekavčić, 1972). Mais la linguistique romane lui doit surtout sa fameuse Grammaire historique de l'italien (Tekavčić, 1972) en trois volumes.

Au cours de ces dernières décennies, la Faculté de philosophie et lettres de Zagreb offre aux étudiants la possibilité de suivre des études complètes de cinq langues et littératures romanes. Parmi les professeurs, certains englobent dans leurs recherches d'autres langues romanes. Dans la section française, c'est le cas de Dražen Varga, qui enseigne essentiellement la linguistique romane ainsi que la linguistique française et publie des travaux sur la syntaxe française et romane en général, mais étudie également les idiomes rhéto-romans. Citons aussi Évaine Le Calvé-Ivičević, qui a introduit dans l'enseignement non seulement l'étude de la traductologie et de la terminologie mais aussi les études canadiennes. Aujourd'hui, d'autres collègues continuent dans ce sillage: ainsi, par exemple, Bogdanka Pavelin Lešić et Darja Damić Bohač étudient les problèmes du français moderne, Karlo Budor ceux de la linguistique espagnole. Nina Lanović fait des recherches sur la lexicologie portugaise et Petar Radosavljević s'efforce de décrire les parlers roumains de Croatie. Au Département de philologie italienne, Smiljka Malinar s'est consacrée à l'étude de l'histoire de la langue italienne, Ivica Peša Matracki a mené, entre autres, des recherches sur la syntaxe italienne, tandis que Maslina Ljubičić et Vinko Kovačić ont travaillé sur la lexicologie italienne etc.

Pour terminer cette présentation, il faut souligner que les linguistes des deux départements s'appliquent, dans leurs recherches, à suivre les méthodes modernes. 


\section{LISTE DES OUVRAGES CITÉS}

Adamović, 1893: Julije Adamović, Francuska gramatika za više razrede srednjih škola, Zagreb (11 éditions jusqu'à 1925).

Adamović, 1901: Julije Adamović, Francusko-hrvatski rječnik, Zagreb, 1901 (trois éditions jusqu'à 1937).

Deanović 1954: Mirko Deanović, Avviamento allo studio del dialetto di Rovigno d'Istria, Zagreb.

Deanović, 1954a; Mirko Deanović, Ricerche sull'istroromanzo. Atti del II Convegno internazionale di linguisti, Milano, 1953.

Deanović-Jernej, 1960: Mirko Deanović, Josip Jernej, Talijansko-hrvatski rječnik, Zagreb.

Deanović-Jernej, 1956: Mirko Deanović, Josip Jernej, Hrvatsko-talijanski rječnik, Zagreb.

Filipović, 1988: Petar Guberina, Filologija, № 15, Zagreb, 1987-1988.

Guberina, 1939: Petar Guberina, Valeur logique et valeur stylistique des propositions complexes en français et en croate, Paris.

Guberina, 1952: Petar Guberina, Zvuk i pokret u jeziku, Zagreb.

Guberina, 1952: Petar Guberina, Povezanost jezičnih elemenata, Zagreb.

Jernej, 1987: Josip Jernej, Talijanska gramatika za početnike i napredne, Zagreb (quatrième édition).

Ježić, 1928: Slavko Ježić, Francuska književnost do kraja klasičnog doba (842-1715), Zagreb.

Kovačec, 1971: August Kovačec, Descrierea istroromânei actuale, Bucureşti.

Kovačec, 1976: August Kovačec, Petar Skok, Etimologijski rječnik hrvatskoga ili srpskoga jezika, Zagreb, I 1971, II 1972, III 1973, IV /Kazalal 1974., Annales de l'Institut français de Zagreb, III, 2 (p. 185-189).

Kovačec, 1993: August Kovačec, Petar Skok (1881-1956), Portreti hrvatskih jezikoslovaca, Biblioteka Hrvatski radio, Knjiga 5, Zagreb. (p. 191-197).

Kovačec 1998: August Kovačec, Istrorumunjsko-hrvatski rječnik (s gramatikom i tekstovima), Pula.

Kovačec, 2005: Josip Jernej (Gorizia, 7 luglio 1909 - Zagabria, 20 marzo 2005). In memoriam. Studia Romanica et Anglica Zagrabiensia, L, Zagreb (p. 221-224).

Kovačec, 2005a: August Kovačec, Jadranske etimologije. Jadranske dopune Skokovu etimologijskom rječniku, Zagreb, I 1998, II 2003, III 2004, Filologija, 44 (p. 117-125).

Kovačec, 2006: Akademik Petar Guberina (22. svibnja 1913. - 22. siječnja 2005.). Spomenica preminulim akademicima, Zagreb (str. 13-23).

Kovačec, 2007: Pavao Tekavčić (Zagabria, 23 agosto 1931 - 19 marzo 2007). In memoriam. Studia Romanica et Anglica Zagrabiensia, LII (p. 337-344).

Kovačec, 2008: Vojmir Vinja (1921. - 2007.). Ljetopis Hrvatske akademije znanosti i umjetnosti za godinu 2007., 111 (p. 517-520).

Kovačec, 2008a: Vojmir Vinja (Dubrovnik, 12. XI. 1921. - Zagreb, 15. VI. 2007.). In memoriam. Studia Romanica et Anglica Zagrabiensia, LIII (p. 379-389).

Kovačec, 2010: August Kovačec, Akademik Vojmir Vinja znanstvenik na nekoliko razboja. Vojmir Vinja 1921. - 2007. Spomenica preminulim akademicima. Zagreb (p. 13-22). 
Kovačec, 2012: August Kovačec, In memoriam Josip Jernej (Gorizia, 7 luglio 1909 - Zagreb, 20 marzo 2005). Zbornik Međunarodnoga znanstvenog skupa u spomen na prof. dr. Josipa Jerneja (1909. - 2005.), Filozofski fakultet, Zagreb, 13. - 14. studenoga 2009., uredili = a cura di Maslina Katušić, Ivica Peša Matracki, Vinko Kovačić, Zagreb, FF press (p. 461-464).

Kovačec, 2013: L'apport scientifique du professeur Vinja dans le domaine de la linguistique romane. Mare loquens. Études d'étymologie et de géolinguistique romanes à la mémoire de Vojmir Vinja (1921-2007), publiées par José Enrique Gargallo et Nikola Vuletić, Zadar, Sveučilište u Zadru (p. 9-22).

Kovačec, 2014: August Kovačec, Akademik Petar Guberina kao romanist = Academician Petar Guberina as a Romanist. Aktualnost Guberinine misli u stoljeću uma= Actuality of Guberina's Thought in the Century of Mind. Znanstveno-stručna monografija VIII. Međunarodnog simpozija verbotonalnog sistema (svibanj 2014.) = Scientific and Professional Monograph of the VIII ${ }^{\text {th }}$ International Symposium of the Verbotonal System (May 2014). U povodu 100. obljetnice rođenja akademika Petra Guberine = Dedicated to Centennial Anniversary of Birth of Academician Petar Guberina, glavna urednica = Adinda Dulčić, Zagreb, Poliklinika SUVAG (str. 99-115).

Muljačić, 1995: Žarko Muljačić, Petar Skok - lingvist, Folia Onomastica Croatica 4, Zagreb (str. 7-17).

Skok, 1906: Petar Skok, Die mit den Suffixen-ācum,-ānum,-āscum und-uscum gebildeten südfranzösischen Ortsnamen. Halle a. S., p. XI+265, Beihefte ZfRPhHeft II.

Skok, 1915: Petar Skok, Pojave vulgarnolatinskoga jezika na natpisima rimske provincije Dalmacije. Djela Jugoslavenske akademije, XXV. P. XII+120, tab. 16.

Skok, 1926, 1930, 1933: Petar Skok, Piccolo contributo allo studio del veglioto, Archivio glottologico italiano, XX, 127-131; XXIV, 19-55; XXV, 117-141.

Skok, 1926a: Petar Skok, Considérations générales sur la déclinaison nominale roumaino-albanaise, Arhiv za arbanasku starinu, jezik i etnografiju, III, 163-175.

Skok, 1927: Petar Skok, Studi toponomastici sull'isola di Veglia, Archivio glottologico italiano, XXI, 95-106 (continué dans les numéros suivants de la revue).

Skok, 1928: Petar Skok, Zum Balkanlatein, ZfRPh XLVIII, 398-413 (continué dans les numéros suivants de la revue).

Skok, 1929: Petar Skok, Studije iz balkanskoga vokabulara, Arhiv za arbanasku starinu, jezik i etnografiju, IV, Beograd, (p. 124-151).

Skok, 1935: Petar Skok, Dolazak Slovena na Mediteran, Split, 1934.

Skok, 1936: Petar Skok, Balkanski jezici (avec Kr. Sandfeld), Knjiga o Balkanu, sv. I, Beograd, (p. 268-275).

Skok, 1936a: Petar Skok, Contribution à l'étude de l'istriote prévénitien, Mélanges dédiés à la mémoire de Prokop M. Haškovec Brno, (p. 310-315).

Skok, 1938: Petar Skok, Pregled francuske gramatike, Zagreb, 1938.

Skok, 1939: Petar Skok, Metodologija francuskog jezika, Zagreb, 1939.

Skok, 1940: Petar Skok, Osnovi romanske lingvistike. I, Kulturno-historijski i opći lingvistički problemi i historijska fonetika naglašenih vokala; II, Historijska fonetika romanskih jezika (Nenaglašeni vokali i konsonanti); III, Historijska morfologija romanskih jezika, Zagreb, 1940. 
Skok, 1943: Petar Skok, Considérations sur le plus ancien istro-roman, Sache, Ort und Wort. Jakob Jud zum sechzigsten Geburtstag 12. Januar 1942. Romanica helvetica vol. 20.; Genève-Zürich, 1942 (p. 472-485., avec une carte).

Skok, 1971, 1972, 1974: Petar Skok, Etimologijski rječnik hrvatskoga ili srpskoga jezika. Uredili Mirko Deanović i Ljudevit Jonke. Surađivao u predradnjama i priredio za tisak Valentin Putanec, Zagreb, I A-J, 1971; II K-poni ${ }^{1}$, 1972; III poni²-Ž, 1973; IV Kazala, 1974.

Spomenica: 1986: Mirko Deanović: 1890-1984. Spomenica preminulim akademicima, Knjiga 32, Zagreb, 1986.

Tekavčić, 1970: Pavao Tekavčić, Uvod u vulgarni latinitet (S izborom tekstova), Zagreb, 1970.

Tekavčić, 1979: Pavao Tekavčić, Uvod u lingvistiku za studente talijanskog jezika, Zagreb, 1979.

Tekavčić, 1972: Pavao Tekavčić, Grammatica storica dell' italiano. Volume I: Fonematica; Volume II: Morfosintassi; Volume III: Lessico. Il Mulino, Bologna, 1972.

Vinja, 1981: Vojmir Vinja, Petar Skok, Studia Romanica et Anglica Zagrabiensia, No 1-2, Zagreb.

Vinja, 1986: Vojmir Vinja, Jadranska fauna. Etimologija i struktura naziva, I-II, Logos-Split.

Vinja, 1998, 2003, 2004 (, 2016): Vojmir Vinja, Jadranske etimologije. Jadranske dopune Skokovu etimologijskom rječniku, Zagreb, Knjiga I, A-H, 1998; Knjiga II, I-Pa, 2003; Knjiga III, Pe-Ž, 2004 Zagreb (Knjiga IV, Kazala, 2016; Sastavili A. Gluhak, O. Ligorio, Nada Vajs Vinja, N. Vuletić).

\section{Sto godina romanske lingvistike na Filozofskom fakultetu Sveučilišta u Zagrebu}

U drugoj polovici 19. st. zagrebačko je sveučilište obnovljeno po uzoru na druga europska sveučilišta i modernizirano pa je od g. 1882. na Mudroslovnome fakultetu bila uvedena praktična nastava francuskoga te uskoro i talijanskoga. No istom je g. 1919. pri Filozofskome fakultetu bio osnovan Odsjek (/Seminar) za romansku filologiju. Prvi profesor romanske lingvistike na tom Odsjeku bio je Skok, nekadašnji student i doktorand Wilhelma Mayera-Lübkea na Sveučilištu u Beču. Skok je započeo sustavno istraživanje romanskih elemenata u hrvatskim govorima jadranske obale i otoka te u govorima balkanskih zemalja. Skoka je naslijedio jedan od njegovih učenika Petar Guberina, koji je u nastavu na zagrebačkoj romanistici uveo Ballyjevu stilistiku, a poslije se sustavno bavio fonetikom i metodama poučavanja stranih jezika. Prvi profesor talijanske filologije, ujedno i osnivač Odsjeka za talijanistiku na Filozofskom fakultetu, bio je Mirko Deanović, koji je posebno proučavao posuđenice iz talijanskoga u hrvatskom pomorskom nazivlju na istočnoj jadranskoj obali te predložio i pokrenuo prikupljanje građe i izradbu Jezičnoga atlasa Sredozemlja. Od godine 1960. Odsjek za romansku filologiju vodio je Vojmir Vinja. On je sustavno proučavao elemente što su ih hrvatski govori obale i otoka posudili u romanskih jezika te toponime romanskoga podrijetla. Profesori Vinja i Deanović poticali su mlađe romaniste, svoje studente, na sustavno proučavanje i opis romanskih idioma koji su se govorili na području Hrvatske i Bosne. Zahvaljujući naporima i poticajima profesora Vojmira Vinje na Filozofskom su fakultetu s vremenom osnovane i organizirane i katedre 
za druge romanske jezike (i književnosti) osim za francuski i talijanski. Na području lingvistike danas se romanistička nastavna i istraživačka djelatnost na Filozofskom fakultetu Sveučilišta u Zagrebu odvija u pet sekcija - francuskoj, španjolskoj, portugalskoj, rumunjskoj (u okviru Odjeka za romanistiku) i talijanskoj (u okviru Odsjeka za talijanistiku).

Ključne riječi: balkanski (jezici), brodogradnja, dalmatski, etimologija, istriotski (istro-romanski), istrorumunjski, kalk, pomorska terminologija, posuđenice, ribarska terminologija, stilistika, toponomastika, venecijanski, židovskošpanjolski 